\title{
A motor timing experiment implemented using a musical instrument digital interface (MIDI) approach
}

\author{
CHARLES E. COLLYER \\ University of Rhode Island, Kingston, Rhode Island \\ SETH S. BOATRIGHT-HOROWTTZ \\ Brown University, Providence, Rhode Island \\ and \\ SARI HOOPER \\ University of Rhode Island, Kingston, Rhode Island
}

\begin{abstract}
The musical instrument digital interface (MIDI) offers a way for perception and behavior researchers to implement high-quality experiments using inexpensive, commercially available hardware and software. We describe the MIDI and illustrate its applicability to research using a replication of the oscillator signature finding reported recently by Collyer, Broadbent, and Church $(1992,1994)$.
\end{abstract}

Many researchers are interested in the psychological and physiological aspects of human motor control, auditory and music perception, time perception, and intrinsic oscillations within the nervous system. Instrumentation in these research areas usually consists of dedicated hardware and software, including a stimulus presentation system, a manipulandum connected to a timer, and an apparatus for storage, analysis, and output. Most systems are dedicated to a single perceptual-motor function and are often expensive or difficult to reconfigure for different types of analyses. In this paper, we describe an experimental control system based on the musical instrument digital interface (MIDI) protocol and provide a description of how a MIDI setup was used to replicate a recent finding in human motor control.

MIDI was developed in the early 1980s (MIDI Manufacturers Association, 1983) as a hardware and software protocol for digital communication. It was quickly adopted by manufacturers of musical instrument technology as a means for allowing rapid, standardized communication among sound generation, control, recording, and computer equipment made by different companies (Huber, 1991; Moog, 1986; Pierce, 1992; Rumsey, 1994).

MIDI data consist of variables describing musical events, such as a note's name, its velocity of activation by a controller key, and its channel assignment. Data are com-

\footnotetext{
The authors thank James A. Kralicky, Arthur A. Little, Neil Massey, Scott Miller, and Dana Scott Mills for their help in conducting the replication study, and especially Russell M. Church and Jonathon Crystal for valuable discussions relating to this article. Correspondence should be addressed to C. E. Collyer, Psychology Department, University of Rhode Island, 10 Chafee Road, Suite 8, Kingston, RI 02881 (e-mail: mgl101@uriacc.uri.edu).
}

municated through unidirectional serial ports labeled MIDI Out, MIDI In, and MIDI Thru. MIDI Out allows the outbound transmission of MIDI information whose destination is the MIDI In port of another unit. MIDI Thru acts as a pass-through for MIDI information that is targeted for the MIDI In ports of other MIDI devices further downstream in a configuration consisting of several devices. A rule of thumb for connecting instruments, controllers, sound cards, computers, and other devices is as follows: MIDI Out to MIDI In to MIDI Thru to MIDI In.

Each MIDI-equipped device is assigned to a channel numbered from 1 to 16 . Channels allow data from a central source, such as sequencer software running on a PC, to address devices without requiring input and output ports for each device. Figure 1 shows a simple MIDI configuration similar to the one used in the experiment described herein.

An experimenter might create a file containing a metronome-like series of notes as follows. A rough approximation to the desired series of notes could be produced on a MIDI controller, such as a keyboard, assigned to one channel. The controller would send event data from its MIDI Out port to the sequencer, where the event data would be recorded on a track. The data on a track can be edited to add or remove notes and to manipulate variations in timing. For example, a quantizing function can be used to regularize the notes so that they occur at precise 250 -msec intervals.

The edited data can be used on another channel to control audio output. The sounds can be played through the output channel with the option of collecting further input on the same track through a controller. Thus, stimulus sounds and response sounds from a subject playing a keyboard or pressing a response key can be added to the 


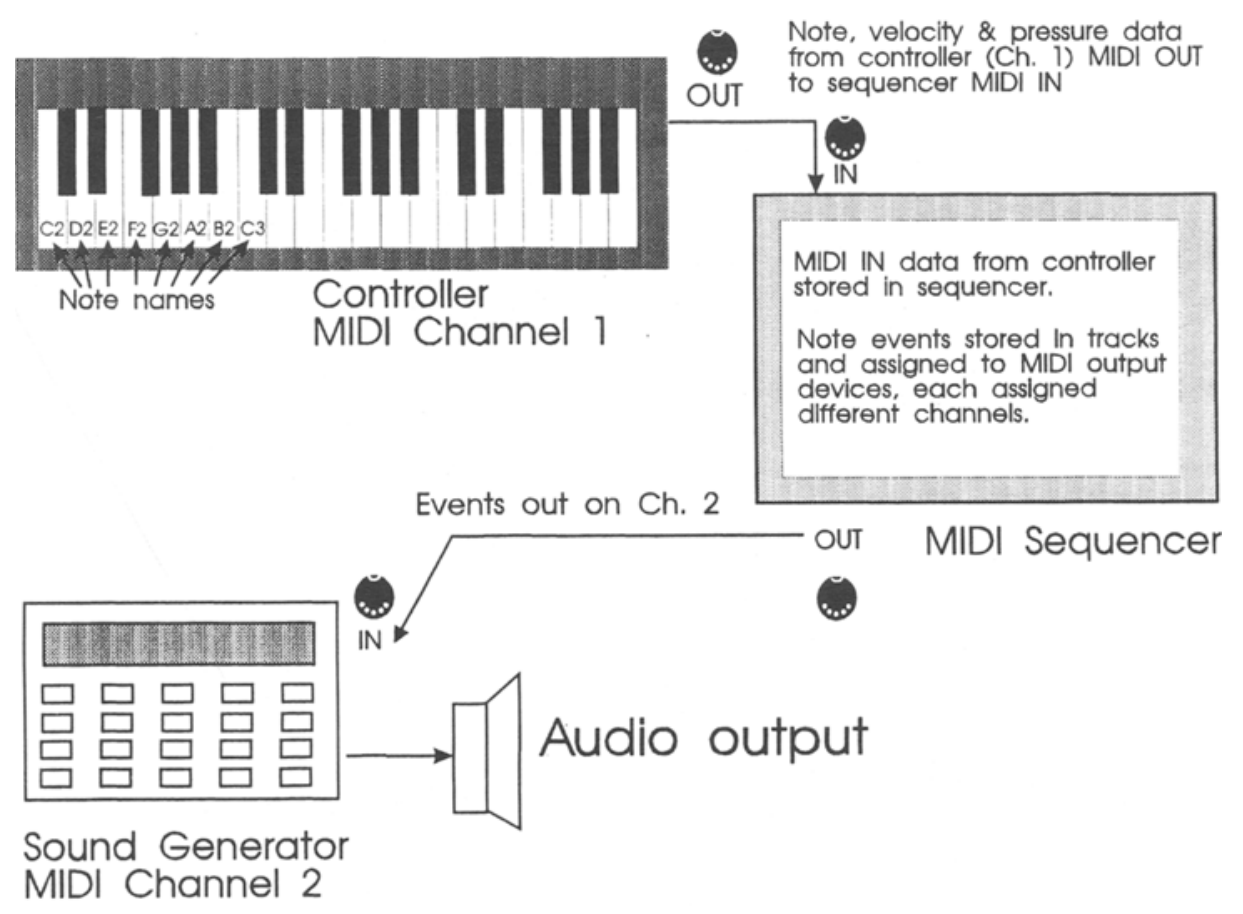

Channel 2 data

activates sound generator

Figure 1. Illustration of musical instrument digital interface (MIDI) terms and relationships. Controllers are input devices such as keyboards and rhythm generators. A sequencer is the software that specifies events (musical note codes) and when they occur. Channels refer to input and output pathways, and devices are assigned to channels. Tracks are components of a music file, most often associated with different instruments.

same or a different track in real time. The complete set of events on all tracks that use the same time line comprises a sequence. A sequence is analogous to the musical score for an orchestra or to an experimenter's protocol for a period of observation and recording of data.

The temporal resolution of a MIDI system is limited to approximately $1 \mathrm{msec}$ and is determined largely by MIDI's serial data transfer rate and 3-byte command length (Kieley, 1991). The temporal jitter, or intercommand variability in real time, of the system will vary with the CPU clock speed, with older and slower computers yielding greater jitter. Some MIDI synthesizer equipment manufactured before 1986 could introduce an event activation delay of several milliseconds, although this is not seen in most current MIDI controllers. Individual system variability can be quantified using various sound analysis packages by sampling the auditory signal from the MIDI output device to a computer sound card input and measuring onset-to-onset time. The measured command resolution of the MIDI system described in this paper was $1.04 \mathrm{msec}$, with jitter of approximately $0.02 \mathrm{msec}$.

Sequencer software stores events in a text fashion that is exportable to spreadsheet, word processing, and statistical software for subsequent analysis. One caveat regarding such exportation of data, however, is that MIDI time events may not be stored in milliseconds, but rather in units that depend on tempo, such as ticks. Tempo is given in beats per minute, and a tick is a subdivision of a beat; in the experiment described herein, we used the greatest temporal precision available, which was 480 ticks per beat or quarter-note. For example, a tempo of 120 implies that a metronome's interstimulus interval (ISI) is $500 \mathrm{msec}$. In general, ISI in milliseconds is equal to 60,000 divided by tempo. The duration of a tick when there are 480 ticks per beat is ISI divided by 480 . Conversion from the measures, beats, and ticks of music to milliseconds is easily carried out with a calculator or spreadsheet.

It is relatively simple both to record input from a MIDI input device and to use a recorded sequence as a schedule for controlling the presentation of stimuli. Figure 2 shows a possible configuration for a MIDI-based operant chamber for use in animal experimentation. A computer running MIDI sequencer software replaces rackmounted control modules for stimulus presentation and data collection. Stimuli can be presented at specified times on the basis of MIDI Out signals from the sequencer. Auditory cues can be presented from an internal sound card or an external MIDI synthesizer. The auditory stimuli can range in complexity from simple tones to any sound sequence that can be recorded or generated. Nonauditory stimuli such as lights can also be presented by converting MIDI Out signals to voltage. MIDI-to- 


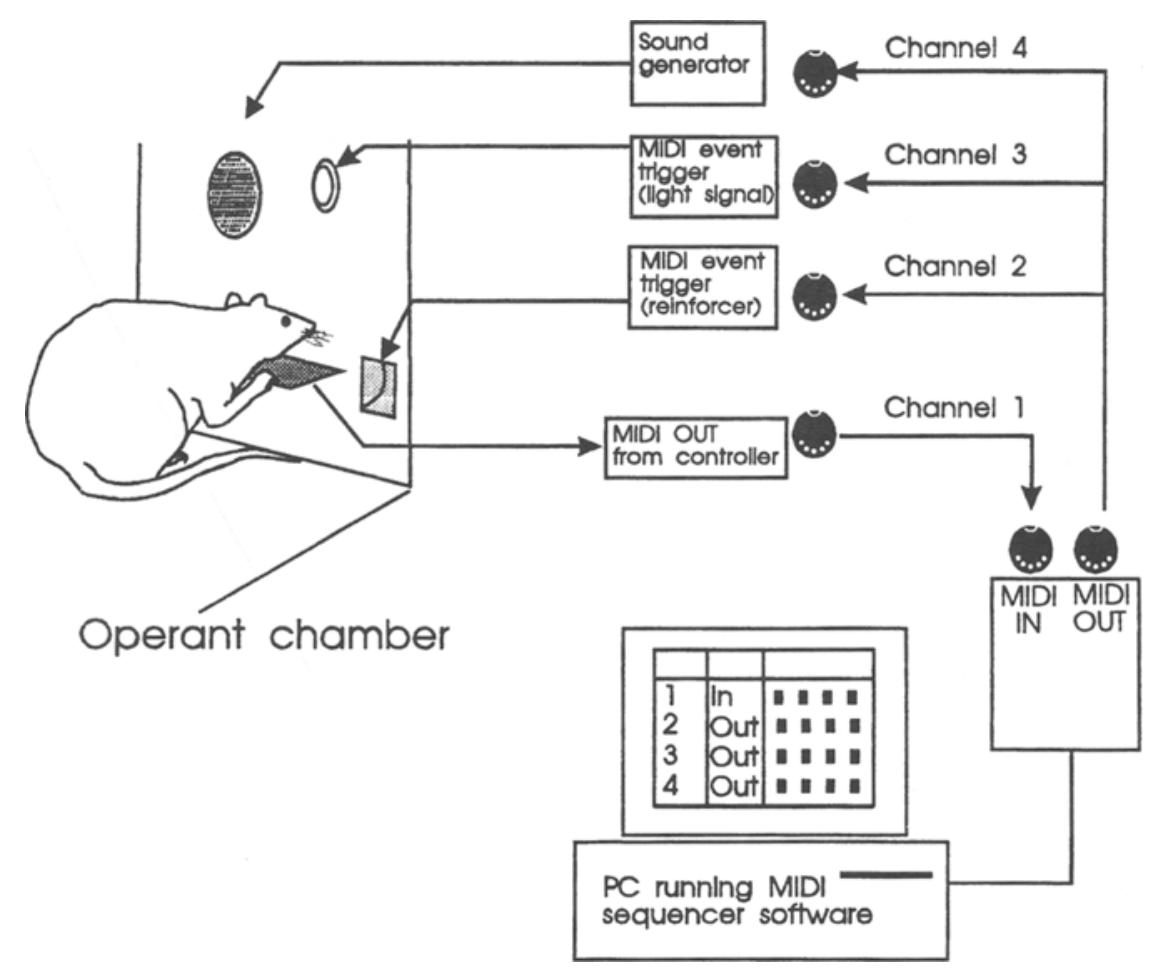

Figure 2. Musical instrument digital interface (MIDI) software and interfacing could be used to control the timing of events and the collection of data in many laboratory situations, such as operant conditioning.

trigger devices are available commercially or can be constructed at low cost. A manipulandum capable of activating a switch or causing a change in voltage can be adapted as a MIDI controller switch and send MIDI data to the MIDI In port, again using commercially available or simply constructed devices.

MIDI hardware and software are available from a large number of sources for systems ranging from high-end audio workstations to simple, older PCs such as the Apple II. Here we describe an inexpensive midrange system using a Soundblaster 16-bit sound card, a MIDIman interface for the sound card, a Casio rhythm generator for input, and Cakewalk Professional music sequencer software. Cakewalk is one sophisticated and flexible software package available for MIDI sequencing; studies similar to the one we describe could also be run using several other sequencer packages.

\section{Example: Replication of the Oscillator Signature}

Collyer, Broadbent, and Church $(1992,1994)$ measured the accuracy of motor timing at several rates of finger tapping. Subjects synchronized finger taps to a metronomelike series of tones in which each tone was temporally separated from the next by an ISI and then continued to tap, attempting to maintain the same ISI, with the tones turned off. There were 28 ISI conditions, ranging from 200 to $875 \mathrm{msec}$ in $25-\mathrm{msec}$ steps. The data of main interest were the interresponse intervals (IRIs) from the continuation part of the trial. Median IRI approximated ISI with good accuracy, but there were small systematic errors in most subjects' performance. Specifically, the function relating IRI (as a percentage: IRI/ISI $\times 100$ ) to ISI was not flat and equal to $100 \%$ except for random error; instead, it was wavelike, with peaks and valleys that departed by only a few percentage points, but significantly, from zero. The term oscillator signature has been applied to this function and to transformed variants of it. ${ }^{1}$

The oscillator signature was the main functional feature of the data to be examined in the replication. Also of interest was the linear increase in variability (measured as standard deviation or semi-interquartile range) with increasing duration that is typically found in timeproduction tasks. The target data for replication were the continuation data of the 16 subjects from the Collyer et al. (1994) study.

Our earlier research on the oscillator signature finding was carried out using IBM AT (in 1992) or PS/2 (in 1994) computers and custom-written experimental software in the Pascal (in 1992) or C (in 1994) languages. The present replication was carried out using entirely different hardware, commercially available MIDI music software, and some macros generated in Excel and QBasic. 


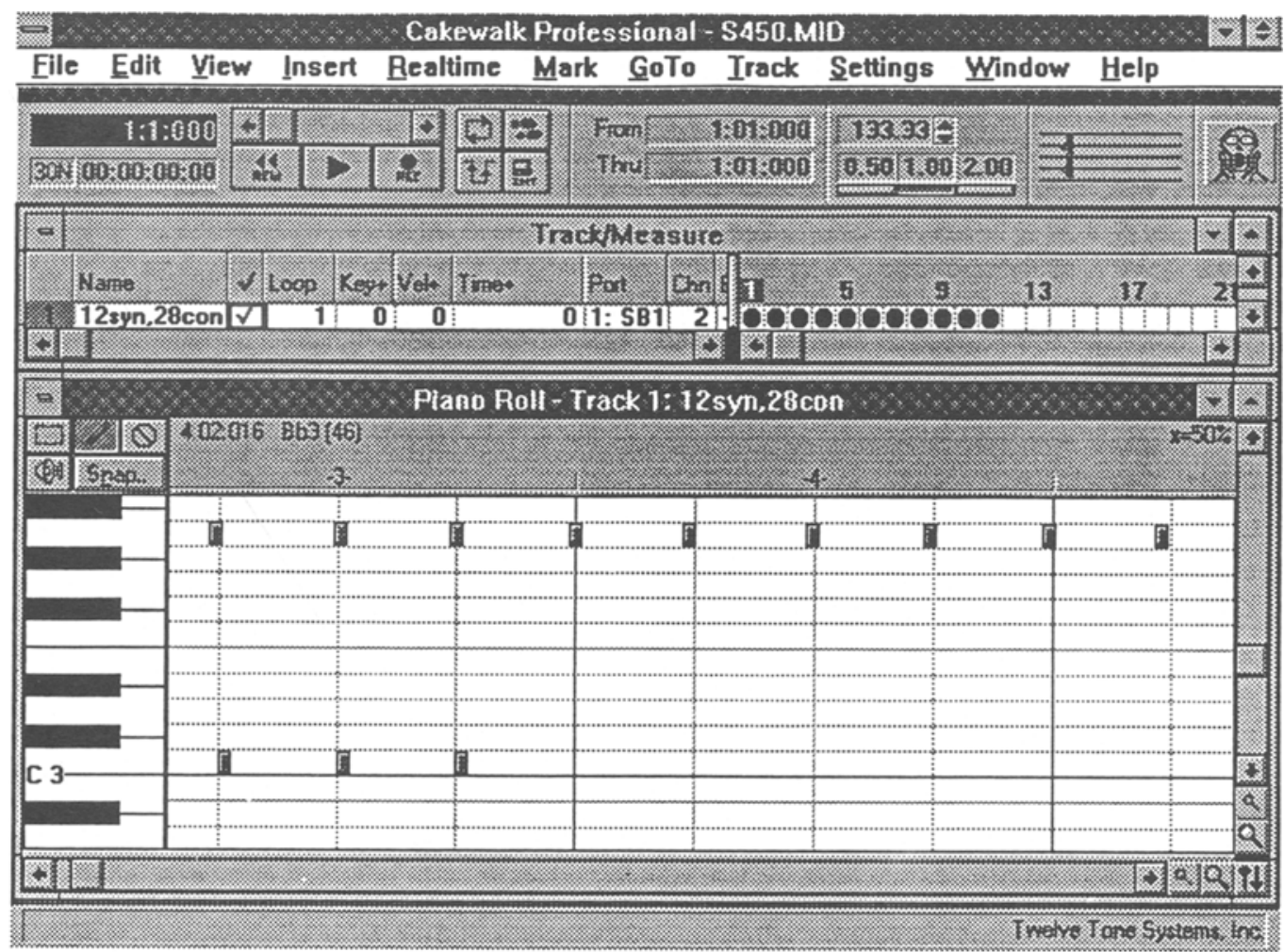

Figure 3. A Cakewalk Version 3.0 screen showing a portion of a tapping trial in the Piano Roll view. Time progresses from left to right. The lower series of note markers shows the last three stimulus sounds before the continuation portion of the trial, and the upper series shows tap responses.

\section{METHOD}

\section{Subjects}

Three graduate students (including Author S.H.), 3 undergraduate students, and a faculty member at the University of Rhode Island (Author C.C.) served as subjects. No subjects served both in this replication and in the target Collyer et al. (1994) study.

\section{Apparatus \\ Hardware consisted of a Gateway 2000 486/DX computer, a Sound Blaster 16 sound card, a MIDIman interface, powered Radio Shack speakers, and a Casio RZ rhythm generator. Software for pre- senting stimuli and collecting responses was the MIDI music se- quencer program Cakewalk Professional, Version 3.0 (Twelve Tone Systems, Inc.). Additional software for file conversion and analy- sis consisted of a shareware program called MF2T for converting MIDI files to ASCII text (van Oostrum, 1995); the commercially available spreadsheet product Microsoft Excel, Version 4.0, for op- erating on text files; and four macros written by members of our lab for streamlining some operations. Macros were checked against the corresponding stepwise manual procedures for accuracy.}

\section{Procedure}

Work files (.WRK files) were prepared in the Cakewalk software for each value of ISI from 175 to $1,000 \mathrm{msec}$ in steps of $25 \mathrm{msec}$. When played, a work file produced a series of 12 sounds (drum notes from the Casio rhythm generator) at the specified ISI. It was necessary to specify a musical tempo in the software that would correspond to each ISI. The precision of this specification varied because tempo is given in beats per minute, and the best resolution of the Cakewalk software is 480 ticks per beat. Consequently, time intervals were accurate to about the nearest millisecond at an ISI of $500 \mathrm{msec}$ (tick duration was $500 / 480=1.04 \mathrm{msec}$ ); precision was better than this at shorter ISIs. At the longest ISI $(1,000 \mathrm{msec})$, intervals were accurate to about the nearest $2 \mathrm{msec}$, which we considered acceptable resolution.

On each trial, the subject first listened to the sounds to hear their tempo and cognitively prepare to reproduce the ISI. The .WRK file was immediately played again in Record mode; subjects attempted to synchronize finger tapping on one of the rhythm generator's control keys with the rate of presentation. The subject continued to tap after the last sound until (after 28 times the ISI interval, or seven musical measures) a distinctive sound signaled the end of the trial. (These trials, consisting of 12 tones and 28 "virtual tones," were shorter than the trials in the 1994 study, which consisted of 50 tones and 50 continuation taps.) At this point, the .WRK file contained both the stimulus sounds and the record of tap responses on a common time line. (A musician would say that a "performance" had been added to the music file.) The file was saved in MIDI code (as a .MID file), and the subject was run on another ISI condition. All 34 ISI conditions were run in this way, in random order, to complete one session lasting about $45 \mathrm{~min}$. Each subject completed three sessions, with no more than one session on any one day.

Figure 3 shows a Cakewalk screen with a .MID file loaded and displayed in the Piano Roll view. The control bar at the top of the screen contains the tempo value 133.33 beats per minute, which specifies the experimental condition ISI $=450 \mathrm{msec}$. The Piano Roll view in the bottom half of the screen shows a portion of the trial history: The three notes to the right of $\mathrm{C} 3$ are the last 3 of the 12 synchronization stimuli; the notes above are the subject's tap responses as synchronization ends and the continuation part of the trial begins. 


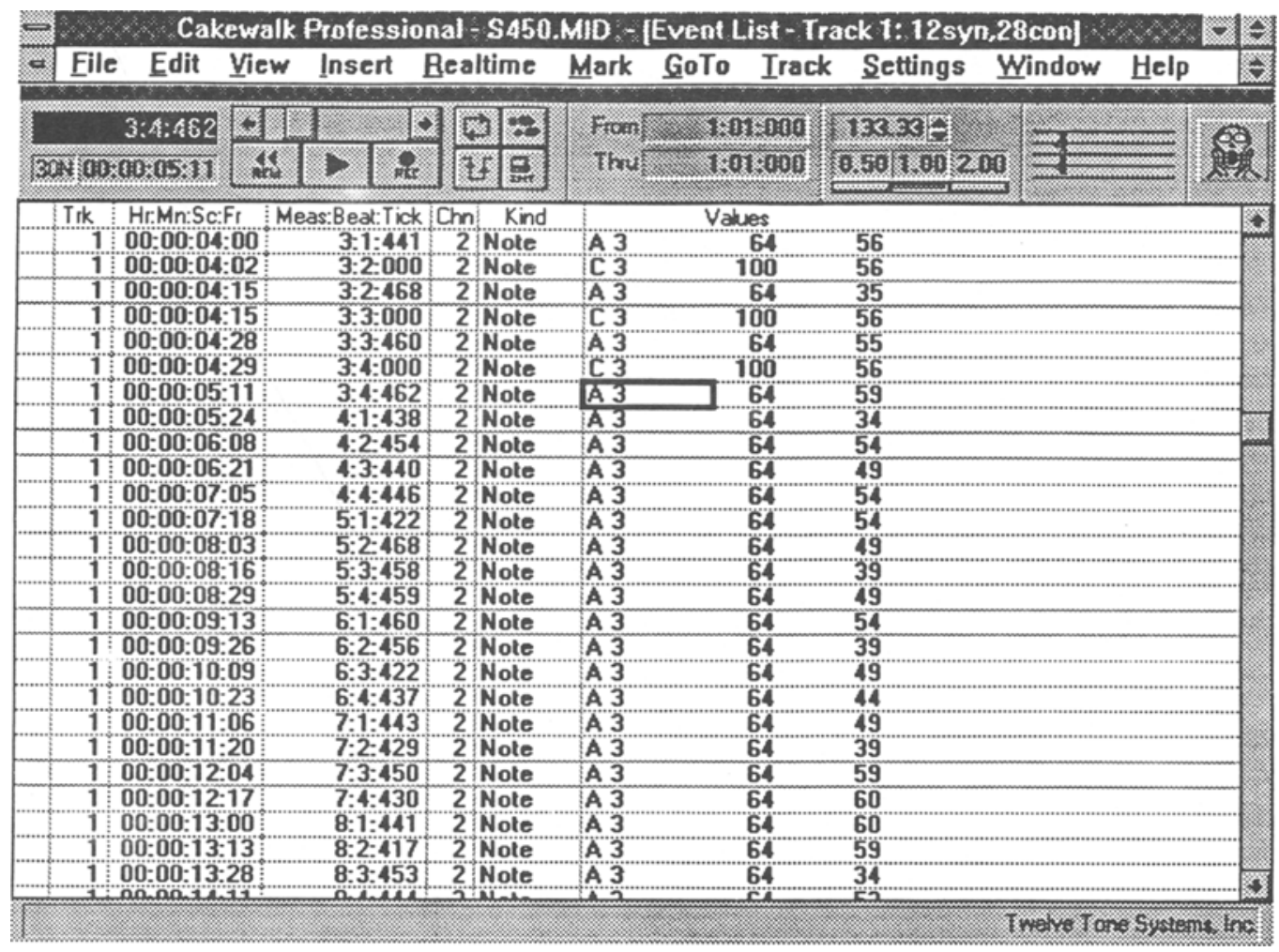

Figure 4. This Cakewalk screen shows the Event List view of the same trial seen in Figure 3. The .MID file containing this information was converted to ASCII, and the series of interresponse intervals was extracted for analysis.

Figure 4 shows a portion of Cakewalk's Event List view of the same trial. The Event List records the time of onset (in both seconds and musical time), the identity (e.g., C3 for stimulus, A3 for response), and the duration (in ticks, in the far-right column) of every event in the file. The highlighted rectangle has been placed on the first continuation tap (the first $\mathrm{A} 3$ after the last $\mathrm{C} 3$ ), corresponding to the fourth response from the left in Figure 3.

Each .MID file was converted to a text file using the MF2T program (van Oostrum, 1995), and then analyzed using Excel macros. The text file was parsed and the stream of successive IRI values was extracted and converted to milliseconds. For each trial, the distribution of IRIs was summarized by calculating the maximum, minimum, and quartile values, as well as the mean and standard deviation. The analyses reported here are based on median IRI values and the semi-interquartile range.

We did not use Cakewalk's programming language in this project; however, this language could provide researchers with additional options for file manipulation and data conversion.

\section{RESULTS AND DISCUSSION}

Figure 5 shows the average oscillator signature of the 16 subjects in the 1994 study and that of the 7 subjects in the present replication. The two functions agree well by visual inspection, and the agreement is confirmed by the correlation of +.78 between them for the set of ISIs where they overlap [Fisher's $z(27)=3.82, p<.001$ ]. This measure of resemblance includes a common linear trend; the linear correlations between the data values and ISI were -.73 for the 1994 data and -.77 for the present replication. The partial correlation of the two data sets, with their common relationship to ISI partialed out, was +.49 [Fisher's $z(27)=2.63, p<.01]$. With or without the linear component of the data pattern, we may say that the oscillator signature has been empirically verified.

Figure 6 shows that a linear regression function provides a good approximation to the increase in variability (indexed by semi-interquartile range), replicating another feature of previous studies. The corresponding function computed from the 1994 data is also shown, highlighting one difference between the two studies. The lower variability in the present replication data may be due to the shorter trials used in the replication experiment. It is possible that in shorter trials, there is less opportunity for drift in the subject's rate of tapping.

Confidence intervals were used by Collyer et al. (1994) to show that the oscillator signature is a statistically reliable departure from perfect accuracy. In the present study, the reliability of the oscillator signature is better demonstrated by the correlation between the original data and the replication. Confidence intervals computed from the present data were comparable in width to those in the earlier analysis, as would be expected from two considerations: (1) The lower variability shown in Figure 6 would decrease the confidence interval at each ISI by a factor of about one third, and (2) the shorter trials of the present study ( 28 taps vs. 50 in the earlier study) would increase the interval by about the same amount. 


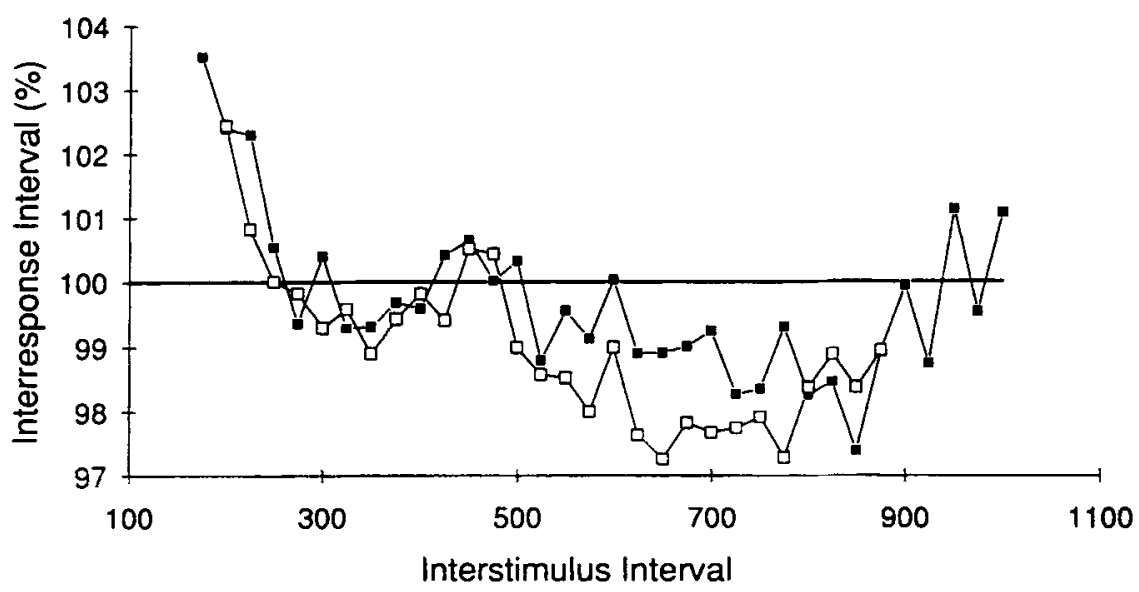

Figure 5. Replication of the oscillator signature finding. The filled data points show average interresponse interval as a percent of interstimulus interval (ISI, in milliseconds) from 175 to 1,000 msec. The open data points show the corresponding data from the Collyer et al. (1994) study for the ISI range of $200-875 \mathrm{msec}$. The close agreement of the two functions validates both the empirical finding of a distinct nonlinearity in human timing and the MIDIbased implementation of the replication experiment.

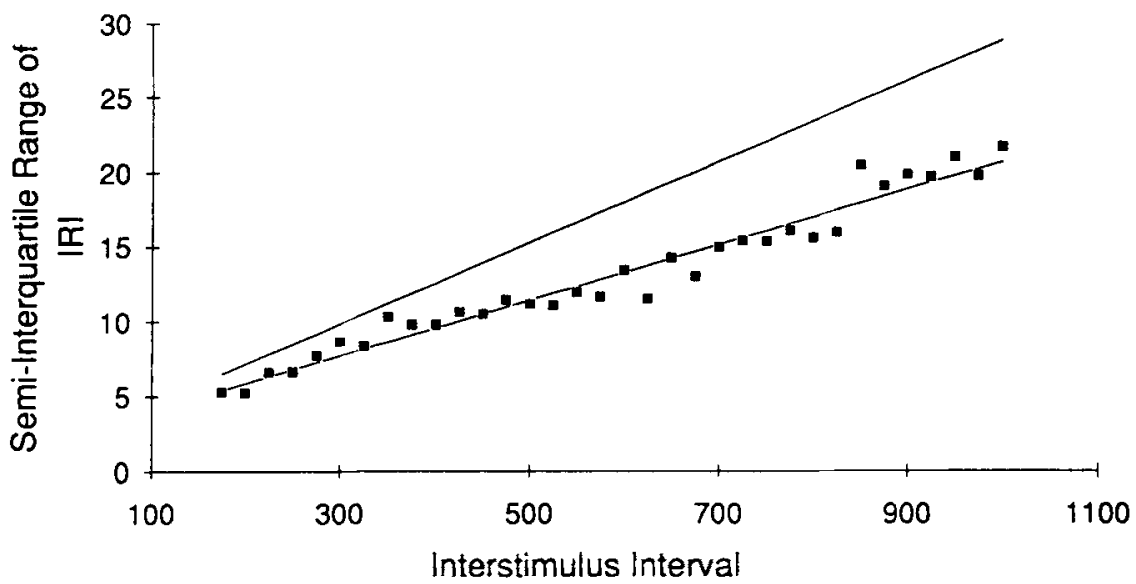

Figure 6. Linear regression function showing the roughly linear increase in variability of interresponse interval (IRI, in milliseconds) as a function of interstimulus interval (also in msec). Data points are from the present replication study and represent the average of 7 subjects' semi-interquartile range estimates. The regression line accounted for $96 \%$ of the variance in these estimates. The corresponding regression line for the data of Collyer et al. (1994) is also shown. The lower variability of the present data could be due to shorter trials ( 28 continuation taps rather than 50), which allow less opportunity for drift.

\section{CONCLUDING REMARKS}

This replication of the oscillator signature encourages further use of a MIDI music approach to implement experiments on timing and time perception, and the primary purpose of this article is to draw attention to MIDI's utility. We began this exercise with the hope that MIDI would prove to be both practical and precise for laboratory work, and it has turned out that way. We also began with the reservation that the musical notation and many unused functions of the Cakewalk software represented a rather inelegant and unwieldy "overhead" in a laboratory control system. However, we have changed our minds on this for a number of reasons. The various graphical representations of events available in Cakewalk have considerable heuristic value. The editing capability of the software brings stimulus specification within the reach of those with more scientific enthusiasm than technical expertise. And the musical overhead actually represents a new domain of experimentation, especially in music perception.

The MIDI-based replication also provides good evidence of the reliability of the oscillator signature finding. Church, Crystal, and Collyer (1996) have recently emphasized the importance of replication for confirming findings and for the correction of possible scientific er- 
rors. The present replication illustrates this point in the context of the authors' own research on timing and time perception. The replication data we have reported here are also featured in a chapter that discusses several issues in the interpretation of continuation tapping data (Collyer \& Church, in press).

\section{REFERENCES}

Church, R. M., Crystal, J. D., \& Collyer, C. E. (1996). Correction of errors in scientific research. Behavior Research Methods, Instruments, \& Computers, 28, 305-310.

Collyer, C. E., Broadbent, H. A., \& Church, R. M. (1992). Categorical time production: Evidence for discrete timing in motor control. Perception \& Psychophysics, 51, 134-144.

Collyer, C. E., Broadbent, H. A., \& Church, R. M. (1994). Preferred rates of repetitive tapping and categorical time production. Perception \& Psychophysics, 55, 443-453.

COLlYer, C. E., \& ChuRCH, R. M. (in press). Interresponse intervals in continuation tapping. In D. A. Rosenbaum \& C. E. Collyer (Eds.), Timing of behavic.r: Neural, psychological, and computational perspectives. Cambridge, MA: MIT Press.

Huber, D. M. (1991). The MIDI manual. Carmel, IN: Sams.

KIELEY, J. M. (1991). MIDI and Macintosh: Searching for a better mousetrap. Behavior Research Methods, Instruments, \& Computers, 23, 256-264.
Midi Manufacturers Association (1983). MIDI I.0 detailed specification. Los Angeles: International MIDI Association.

MoOG, R. (1986). MIDI: The musical instrument digital interface. Journal of the Audio Engineering Society, 34, 394-404.

Pierce, J. R. (1992). The science of musical sound (rev. ed.). New York: W. H. Freeman.

RUMSEY, F. (1994). MIDI systems and control (2nd ed.). Oxford, U.K.: Focal Press.

VAN Oostrum, P. (1995). MF2T. [Program to convert MIDI files to ASCII. Shareware accessed by Internet.]

\section{NOTE}

1. In earlier published work (Collyer et al., 1992, 1994), the data analysis included linear regression of each subject's IRI on ISI, and the oscillator signature was defined in terms of variation around this line. In the present study, the regression step was omitted, and the oscillator signature was defined in terms of variation around the identity function $($ IRI = ISI). There are advantages and disadvantages to each procedure; the approach used in this paper was chosen for its simplicity and because the theoretical implications of individual normalization are not of interest here. A consequence of this procedural difference is that the 1994 oscillator signature has a linear trend in Figure 5 of this paper, but does not in Figure 4 of Collyer et al. (1994).

(Manuscript received February 12, 1996; revision accepted for publication July $1,1996$. 